\title{
Positional uncertainty of network RTK observations in a modern datum
}

DOI: https://doi.org/10.1515/jogs-2020-0116

Received November 9, 2020; accepted March 29, 2021

\begin{abstract}
The Geocentric Datum of Australia 2020 (GDA2020) is Australia's new and much improved national datum. It is based on a single, nationwide least squares network adjustment that rigorously propagates uncertainty. This paper explores three options to include Network Real-Time Kinematic (NRTK) observations and their Positional Uncertainty (PU) in the survey control network of New South Wales (NSW) via the GDA2020 state adjustment. In the first option, PU is empirically estimated based on a dataset of more than 1,500 observations to obtain values that can be uniformly applied to all NRTK observations. In the second option, PU is calculated for each NRTK observation, based on the coordinate quality indicators provided by the Global Navigation Satellite System (GNSS) equipment. Both options continue to treat NRTK observations as point-based position solutions, resulting in poor correlation with surrounding survey control marks. The third option overcomes this issue by utilising the automatically computed GNSS baselines between NRTK observations and their Virtual Reference Station (VRS) to create a connected network that can be adjusted like a static GNSS network. Using a typical urban NRTK survey in Sydney as an example, it is shown that this method offers a rigorous computation of PU, while maintaining the quick and easy nature of NRTK positioning.
\end{abstract}

Keywords: Datum modernisation, national datum, network adjustment, network-based positioning, Positional Uncertainty, Virtual Reference Station

\section{Introduction}

The Geocentric Datum of Australia 2020 (GDA2020) is Australia's new and much improved national datum, introduced in October 2017 and adopted in New South Wales

\footnotetext{
T. Bernstein: Spatial Services, NSW Department of Customer Service, Sydney, Australia

*Corresponding Author: V. Janssen: Spatial Services, NSW Department of Customer Service, Bathurst, Australia, E-mail: Volker.Janssen@customerservice.nsw.gov.au
}

(NSW) on 1 January 2020. This modern datum is defined in the International Terrestrial Reference Frame 2014 (ITRF2014, Altamimi et al. 2016) at epoch 2020.0 and based on a single, nationwide least squares network adjustment that rigorously propagates uncertainty (ICSM 2020a).

Spatial Services, a business unit of the NSW Department of Customer Service (DCS), is responsible for the maintenance and extension of the State's survey control network, which comprises 250,000 survey marks on public record. The backbone of the NSW survey control network is provided by CORSnet-NSW, Australia's largest stateowned and operated Global Navigation Satellite System (GNSS) Continuously Operating Reference Station (CORS) network. CORSnet-NSW currently consists of 200 stations, providing fundamental positioning infrastructure that is authoritative, accurate, reliable and easy-to-use for a wide range of applications (e.g. Janssen 2012; Janssen et al. 2016; NSW Spatial Services 2021).

At present, the GDA2020 state adjustment incorporates approximately 43,300 survey control marks across NSW, i.e. $17 \%$ of the 250,000 marks on public record. Consequently, $83 \%$ of the marks have been transformed from the now superseded GDA94 to GDA2020. Uncertainties of these transformed GDA2020 coordinates cannot be computed until the underlying measurements are sourced and readjusted with a well-defined connection to datum in the GDA2020 state adjustment.

NSW Spatial Services is accelerating the process of including additional survey marks into the state adjustment in order to improve user access to GDA2020 coordinates and uncertainties (Janssen and McElroy 2020). Given that Network Real-Time Kinematic (NRTK) observations are generally treated as point-based position solutions, it is necessary to investigate how to assign realistic uncertainties that can be incorporated into the GDA2020 state adjustment. When using CORSnet-NSW, single-base RTK positioning results can be expressed as a baseline to the CORS used and thus ingested. However, while NRTK has been shown to provide superior positioning quality compared to single-base RTK and is therefore preferable (e.g. Edwards et al. 2010; Wang et al. 2010; Janssen and Haasdyk 2011), this process is not as straight-forward. 
Positional Uncertainty (PU) is defined as the uncertainty of the horizontal and/or vertical coordinates of a point, at the 95\% confidence level (CL), with respect to the defined datum (ICSM 2020b). It can be separated into Horizontal PU (HPU) for horizontal position and Vertical PU (VPU) for ellipsoidal height. HPU is expressed as the radius of a $95 \%$ circle of uncertainty, generally calculated from the standard error ellipse produced by a least squares network adjustment. VPU is a linear quantity and obtained by scaling the standard deviation by 1.96 to convert it to $95 \%$ confidence. A description of the practical implementation of PU on public record in NSW can be found in Janssen et al. (2019).

This paper explores three options to include NRTK observations and their uncertainties in the NSW survey control network via the GDA2020 state adjustment. In the first option, the feasibility of empirically estimating the PU of NRTK observations is investigated, although it is acknowledged that it is ultimately desired to compute uncertainties, rather than estimate them. In the second option, in an effort to obtain PU values more tailored to each individual occupation, the reliability of using coordinate quality indicators provided by the GNSS equipment to calculate PU on an occupation-by-occupation basis is explored. However, this computation requires input of the PU of the reference station used, which is generally a Virtual Reference Station (VRS, Landau et al. 2002) for NRTK with CORSnetNSW, with the PU of a VRS not yet suitably quantified.

These two options continue to treat NRTK observations as point-based position solutions, which results in a lack of correlation with the surrounding network. The third option investigated overcomes this issue by utilising the automatically computed GNSS baselines between NRTK observations and their VRS to create a connected network, which can be adjusted like a static GNSS network. It is shown that this offers a rigorous computation of $\mathrm{PU}$, while maintaining the quick and easy nature of NRTK positioning.

\section{Empirical estimate of NRTK PU}

The first option to incorporate NRTK uncertainties into the GDA2020 state adjustment was to determine an empirical estimate of PU that can be applied to all NRTK observations. To this end, a large and robust historical NRTK dataset collated from several projects conducted by NSW Spatial Services was examined. Data was collected under a range of observing conditions typically encountered in surveying practice, with various NRTK cell sizes, dif- ferent instruments and including centring errors, providing a suitable indication of real-world NRTK performance. Best practice guidelines were followed, including minimum observation times of 2 minutes (windowing technique) and double (or more) occupations at least $30 \mathrm{~min}$ utes apart (ICSM 2020b; NSW Spatial Services 2019).

The collated data was prepared based on the following criteria to remove large outliers in order to obtain a realistic and representative estimate of NRTK PU in practice:

- Only marks with two or more occupations were used for comparison.

- Only observations with successful ambiguity resolution were retained, here defined by a coordinate quality (CQ) value of below $0.050 \mathrm{~m}$.

- Marks showing a horizontal coordinate difference larger than $0.060 \mathrm{~m}$ between two occupations were removed. This value was chosen based on the standard deviation routinely applied by NSW Spatial Services for horizontal NRTK uncertainty using CORSnet-NSW in practice $(0.014 \mathrm{~m})$, by rounding up to $0.015 \mathrm{~m}$ and multiplying by 4 .

- Marks showing a vertical coordinate difference larger than $0.120 \mathrm{~m}$ between two occupations were removed. This value was chosen based on the standard deviation routinely applied by NSW Spatial Services for vertical (ellipsoidal height) NRTK uncertainty using CORSnet-NSW in practice $(0.030 \mathrm{~m})$ and multiplying by 4 .

After preparation, the dataset consisted of 1,535 observations on 756 marks across eastern NSW (Fig. 1). The differences in horizontal coordinates and ellipsoidal height between double (or more) occupations on each mark were determined and analysed. It is recognised that this provides a measure of NRTK precision (repeatability) rather than accuracy with respect to datum. However, the Root Mean Square (RMS), at the 95\% confidence level, of these coordinate differences is similar to a measure of PU and deemed a suitable approximation for everyday users.

The differences between multiple NRTK occupations on the same mark in horizontal position and ellipsoidal height were examined to determine an empirical, realworld estimate of PU. Histograms of these differences (799 in total) are shown in Fig. 2, while descriptive statistics (minimum, maximum, mean, median, standard deviation and RMS) are summarised in Table 1.

It is recognised that the PU values in the GDA2020 state adjustment are not normally distributed, but instead present as a skewed, right-tailed distribution (Janssen et al. 2019). For such a skewed distribution, the median provides a more robust measure of central tendency than the 


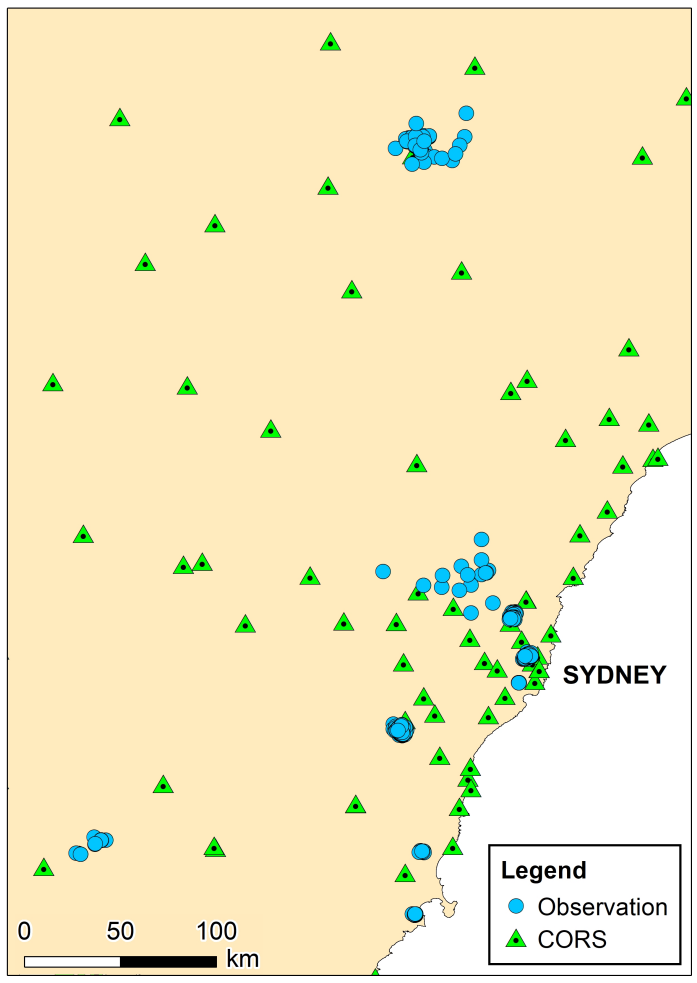

Fig. 1. Location of CORSnet-NSW sites and NRTK data collected across eastern NSW.
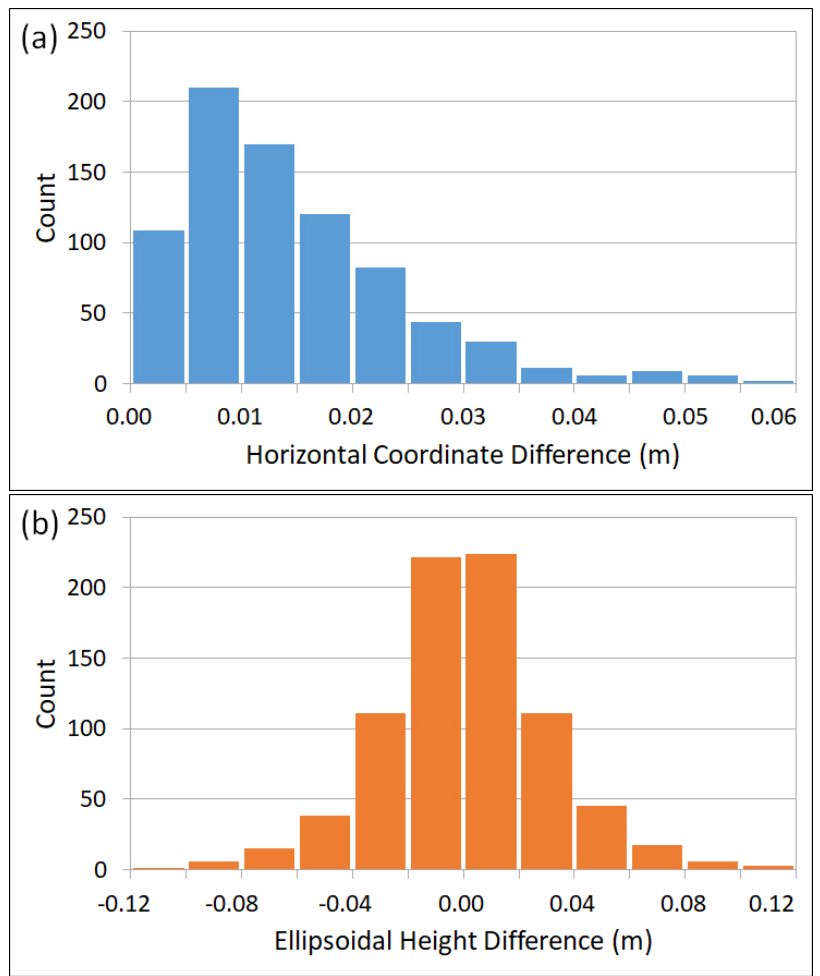

Fig. 2. (a) Horizontal coordinate differences and (b) ellipsoidal height differences between multiple NRTK occupations.
Table 1. Descriptive statistics for 799 horizontal distances and ellipsoidal height differences between multiple NRTK occupations.

\begin{tabular}{ccc}
\hline Descriptive Statistic & Horiz. $(\mathrm{m})$ & Vert. $(\mathrm{m})$ \\
\hline Minimum & 0.000 & -0.106 \\
Maximum & 0.060 & 0.108 \\
Mean & 0.015 & 0.001 \\
Median & 0.013 & 0.001 \\
Standard Deviation & 0.010 & 0.030 \\
RMS at 95\% CL & 0.036 & 0.059 \\
\hline
\end{tabular}

mean and is less susceptible to outliers. In this paper, both the mean and median values are shown to illustrate their agreement and that a normal distribution is suitable for the analysis presented here.

The horizontal data shows a normal distribution with a slight positive, right-tailed skew, producing an RMS value of $0.036 \mathrm{~m}$ at the $95 \%$ confidence level. Using a normal approximation, one can estimate that $95 \%$ of NRTK observations have horizontal coordinate differences below two standard deviations from the mean, i.e. below $0.035 \mathrm{~m}$ in this case. Given the slight skewness of the distribution, the $95^{\text {th }}$ percentile was also calculated as a method of verification, returning $0.034 \mathrm{~m}$ and therefore showing good agreement. The vertical data provides an RMS of $0.059 \mathrm{~m}$ (95\% CL). Using a normal approximation, it is estimated that $95 \%$ of ellipsoidal height differences are within 0.061 $\mathrm{m}$, and the $95^{\text {th }}$ percentile (based on absolute height differences) returns $0.063 \mathrm{~m}$ to support this value.

In order to verify the results of this analysis, findings were compared to a previous, extensive study quantifying the performance of NRTK across NSW at various distances from the surrounding CORS (Janssen and Haasdyk 2011). The achievable precision was investigated over three consecutive days using multiple GNSS receivers at four different locations, while the achievable accuracy was determined by comparison to the NSW survey control network in seven test areas exhibiting a range of NRTK scenarios and cell sizes.

Table 2 summarises the precisions achieved for 2minute NRTK solutions over a range of distances from the surrounding CORS, expressed as RMS and converted to 95\% CL to aid comparison. In addition, Janssen and Haasdyk (2011) quantified the achievable NRTK accuracy in GDA94 (based on 1-minute observation windows) as about $0.040 \mathrm{~m}$ (or better) in the horizontal and $0.060 \mathrm{~m}$ in the vertical component, again expressed as RMS at 95\% CL, provided that recommended inter-CORS distances are used.

Considering the densification of CORSnet-NSW since 2011 (Janssen et al. 2016), most of the data in the present study was collected in smaller NRTK cells, with the largest 
Table 2. Precisions for NRTK solutions (2-minute observation windows) for a range of NRTK cell sizes (Janssen and Haasdyk 2011).

\begin{tabular}{cccc}
\hline Distance to Nearest CORS (km) & 6 & 15 & 50 \\
\hline Horizontal RMS at 95\% CL (m) & 0.012 & 0.024 & 0.041 \\
Vertical RMS at 95\% CL (m) & 0.020 & 0.037 & 0.110 \\
\hline
\end{tabular}

distance to the nearest CORS being $37 \mathrm{~km}$. The empirically estimated values of $0.036 \mathrm{~m}$ (HPU) and $0.059 \mathrm{~m}$ (VPU) are therefore deemed comparable to the values stated in Janssen and Haasdyk (2011). It should be noted that the previous study was performed under more controlled conditions, so the effects of challenging observing conditions and centring errors had less impact than on the data presented here.

In summary, this approach utilises historical data to assess real-world NRTK performance, providing empirically derived estimates of $0.036 \mathrm{~m}$ for HPU and $0.059 \mathrm{~m}$ for VPU. This simplistic method can be easily applied to all NRTK observations, including historical NRTK data. However, major limitations are that it provides estimated (rather than rigorously calculated) uncertainties and continues to treat NRTK observations as point-based position solutions, thus exhibiting poor correlation with surrounding marks. It is also acknowledged that these values may not always be realistic, particularly under challenging observing conditions. Whilst ICSM (2020b) allows authorities to empirically estimate PU, NSW Spatial Services has adopted a more rigorous approach and only publishes calculated PU values on public record. Hence, this method is not suitable if this philosophy is to be maintained.

\section{Computing PU based on coordinate quality (CQ) output}

The second option to introduce NRTK uncertainties into the GDA2020 state adjustment attempted to reflect the individual qualities of each NRTK observation in the computation of PU on an occupation-by-occupation basis, thereby introducing more rigor into its determination. Therefore, the feasibility of using coordinate quality (CQ) indicators provided by the GNSS equipment was explored. CQ is usually calculated at the rover as the RMS of coordinate errors, generally based on ambiguity-fixed, doubledifferenced observations, and indicates how much the computed position is likely to deviate from the 'true' value.

Research has shown that CQ values are prone to be overly optimistic, especially under difficult observing conditions (e.g. Edwards et al. 2010; Wang et al. 2010; Janssen and Haasdyk 2011), hence this approach needs to be treated with caution. The reliability of CQ values was assessed, along with the possibility of quantifying the disparity between quoted CQ values and the actual quality observed through a scale factor to account for exaggerated CQ reporting.

The method used to compute PU for NRTK observations was adapted from the Queensland Government's cadastral survey requirements (Queensland Government 2020). Instead of using manufacturer specifications to estimate measurement uncertainty, the CQ output was used (i.e. standard deviation in Easting, Northing and ellipsoidal height). Findings were compared to the NRTK data presented in section 2 to determine how well the computed values match real-world results.

In the horizontal component, Measurement Uncertainty (MU) is computed by the law of propagation of errors from the standard deviations in Easting and Northing, multiplied by a coverage factor of 2.45 (for a 2dimensional quantity) to obtain values at the $95 \%$ confidence level (ICSM 2020b):

$$
M U_{h z}=\sqrt{\sigma_{E}^{2}+\sigma_{N}^{2}} \star 2.45
$$

The Survey Uncertainty (SU) of the mean of two observations A and B on the same mark is given by ( $n=$ number of observations, i.e. in this case $n=2$ ):

$$
S U_{\text {mean }}=\sqrt{\frac{M U_{A}^{2}+M U_{B}{ }^{2}}{n^{2}}}
$$

$\mathrm{PU}$ is then calculated by considering the rover's centring error $\left(E_{\text {cent }}\right)$ and the uncertainty of the reference station $\left(P U_{r e f}\right)$ :

$$
P U=\sqrt{S U_{\text {mean }}^{2}+E_{\text {cent }}{ }^{2}+P U_{\text {ref }}^{2}}
$$

The reference station is usually a VRS for NRTK observations with CORSnet-NSW, and the PU of a VRS is not readily available, so an estimate is required. PU values for CORS, typically around $0.010 \mathrm{~m}$, can be used as a guide. In this case, a VRS is assumed to have a larger PU of $0.020 \mathrm{~m}$, and a nominal centring error of $0.004 \mathrm{~m}$ for the rover is applied.

In the vertical component, $\mathrm{MU}$ is calculated using a coverage factor of 1.96 to convert the standard deviation of ellipsoidal height as a 1-dimensional quantity to the $95 \%$ confidence level (ICSM 2020b):

$$
M U_{h}=\sigma_{h} \star 1.96
$$

$S U_{\text {mean }}$ is then calculated via Eq. (2), and VPU follows using Eq. (3). In this case, the VRS is assumed to have a VPU of $0.040 \mathrm{~m}$, and a nominal centring (or instrument height) error of $0.004 \mathrm{~m}$ for the rover is used. 
Applying this procedure and the assumed parameters to our dataset produced the results illustrated in Fig. 3. Corresponding descriptive statistics are summarised in Table 3.
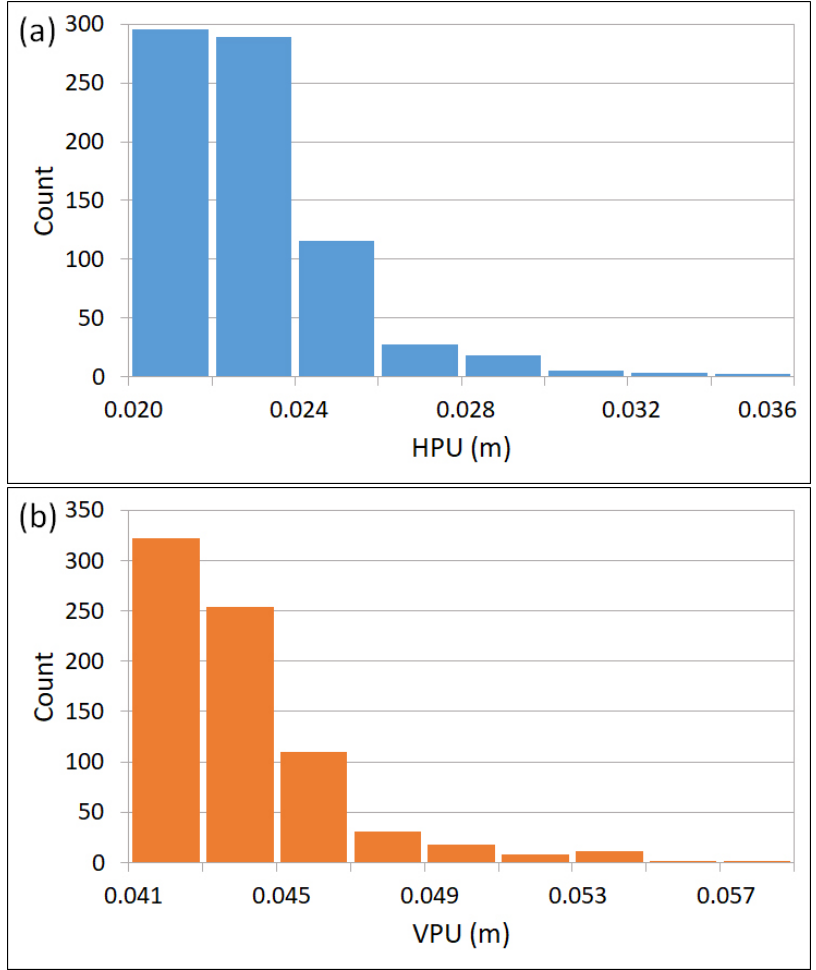

Fig. 3. Calculated (a) HPU and (b) VPU for NRTK observations based on CQ output.

Table 3. Descriptive statistics for HPU and VPU, calculated based on CQ output for 756 marks.

\begin{tabular}{ccc}
\hline Descriptive Statistic & HPU $(\mathrm{m})$ & VPU $(\mathrm{m})$ \\
\hline Minimum & 0.021 & 0.041 \\
Maximum & 0.036 & 0.057 \\
Mean & 0.023 & 0.044 \\
Median & 0.023 & 0.043 \\
Standard Deviation & 0.002 & 0.002 \\
RMS & 0.024 & 0.044 \\
\hline
\end{tabular}

All 756 marks have calculated HPU values within the empirical estimate of $0.036 \mathrm{~m}$ obtained from the same dataset, with a median HPU of $0.023 \mathrm{~m}$ and a $95^{\text {th }}$ percentile of $0.027 \mathrm{~m}$. In the vertical, all values are significantly less than the estimated VPU of $0.059 \mathrm{~m}$, with a median of $0.043 \mathrm{~m}$ and a $95^{\text {th }}$ percentile of $0.049 \mathrm{~m}$. In other words, 585 marks (i.e. 77\%) have a calculated HPU of 0.024 $\mathrm{m}$ or better, while 576 marks (i.e. $76 \%$ ) have a calculated
VPU of $0.045 \mathrm{~m}$ or better. The clearly optimistic nature of NRTK PU calculated by this method can be attributed mainly to using unrealistic CQ values. While the need to estimate the PU of the VRS is also acknowledged as a possible cause, the estimates chosen are rather conservative based on NSW Spatial Services' experience of using NRTK in practice.

\subsection{Scaling CQ values}

Several studies (e.g. Edwards et al. 2010; Wang et al. 2010; Janssen and Haasdyk 2011) have shown CQ values to be rather unrealistic in practice, being overly optimistic by a factor of up to 5-7. This is in part due to the CQ calculation not considering external errors such as multipath or centring errors. It would be beneficial for the user to have the option of specifying values for some of these errors in the GNSS rover software, which could result in more realistic $\mathrm{CQ}$ values.

Using the NRTK data collected, the feasibility of modelling the difference between the quoted CQ value and the measured precision was examined. This can potentially provide a scale factor to be applied to the above calculation, thereby aligning the computed PU with the observed precision of real-world NRTK observations. In order to assess the reliability of the reported CQ values, the ratio of the horizontal distance (or the absolute ellipsoidal height difference) between reoccupations on each mark to the horizontal (or vertical) CQ output was computed for each occupation (Fig. 4 and Table 4).

Table 4. Descriptive statistics for measured vs. reported quality ratios.

\begin{tabular}{ccc}
\hline Descriptive Statistic & Horizontal & Vertical \\
\hline Minimum & 0.02 & 0.01 \\
Maximum & 9.32 & 9.32 \\
Mean & 2.38 & 1.96 \\
Median & 2.09 & 1.55 \\
Standard Deviation & 1.49 & 1.62 \\
\hline
\end{tabular}

As expected, most reported CQ values appear to be overly optimistic as evident by a coordinate quality ratio larger than 1 . In the horizontal component, $84 \%$ of the 1,535 occupations delivered overly optimistic CQ values, with a median ratio of about 2.1. In the vertical, $65 \%$ of $C Q$ values were overly optimistic, with a median ratio of about 1.6. The mean values are larger than the median, indicating that the dataset is affected by several outliers. A substantial number of observations produce $C Q$ values that are op- 

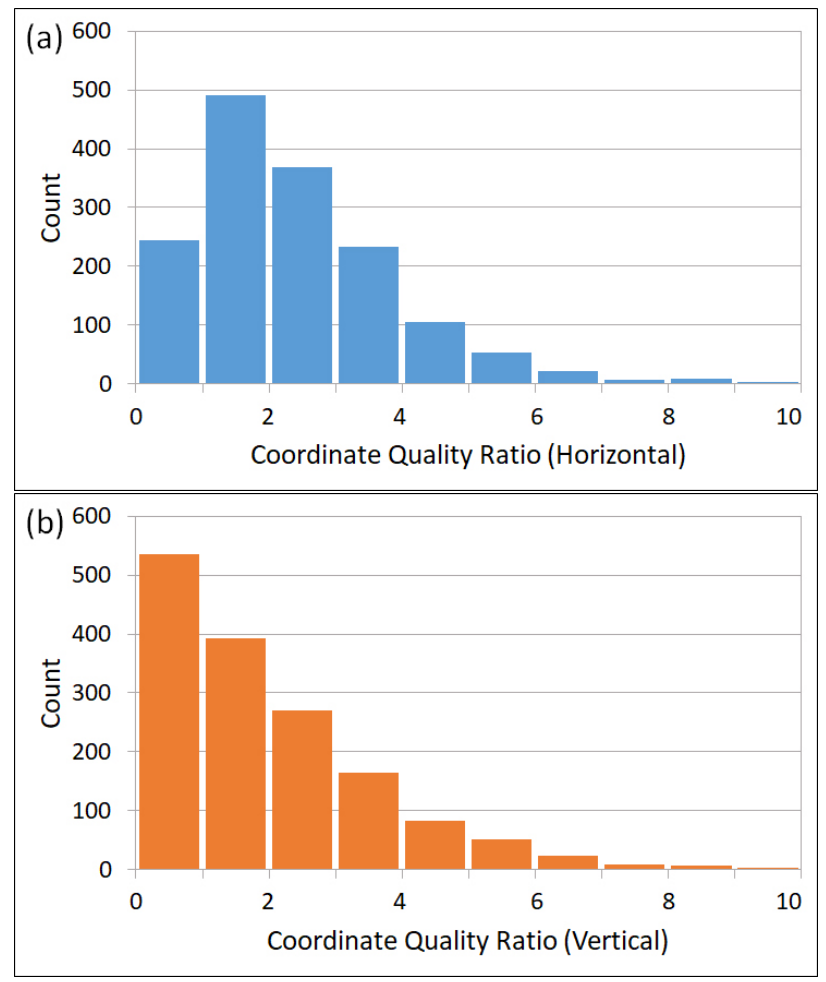

Fig. 4. Ratio of measured coordinate quality to reported CQ output for the (a) horizontal and (b) vertical components.

timistic by more than a factor of 4 (and up to a factor of 9 ) in both components. This is particularly of concern when attempting to determine a reasonable and reliable scale factor in order to calculate PU for NRTK observations with a sufficient level of rigor.

Interestingly, and unexpectedly, the vertical $C Q$ values appear to be more realistic than the horizontal CQ values for the data investigated. Being conservative, a scale factor of 2.1 was applied to both the horizontal and vertical CQ values in Eq. (1) and Eq. (4), respectively, to obtain more realistic $\mathrm{PU}$ values. This produced the results shown in Fig. 5, with descriptive statistics summarised in Table 5.

Table 5. Descriptive statistics for HPU and VPU, calculated based on CQ output scaled by a factor of 2.1 for 756 marks.

\begin{tabular}{ccc}
\hline Descriptive Statistic & HPU $(\mathrm{m})$ & VPU $(\mathrm{m})$ \\
\hline Minimum & 0.022 & 0.042 \\
Maximum & 0.066 & 0.093 \\
Mean & 0.032 & 0.053 \\
Median & 0.030 & 0.051 \\
Standard Deviation & 0.006 & 0.008 \\
RMS & 0.032 & 0.054 \\
\hline
\end{tabular}
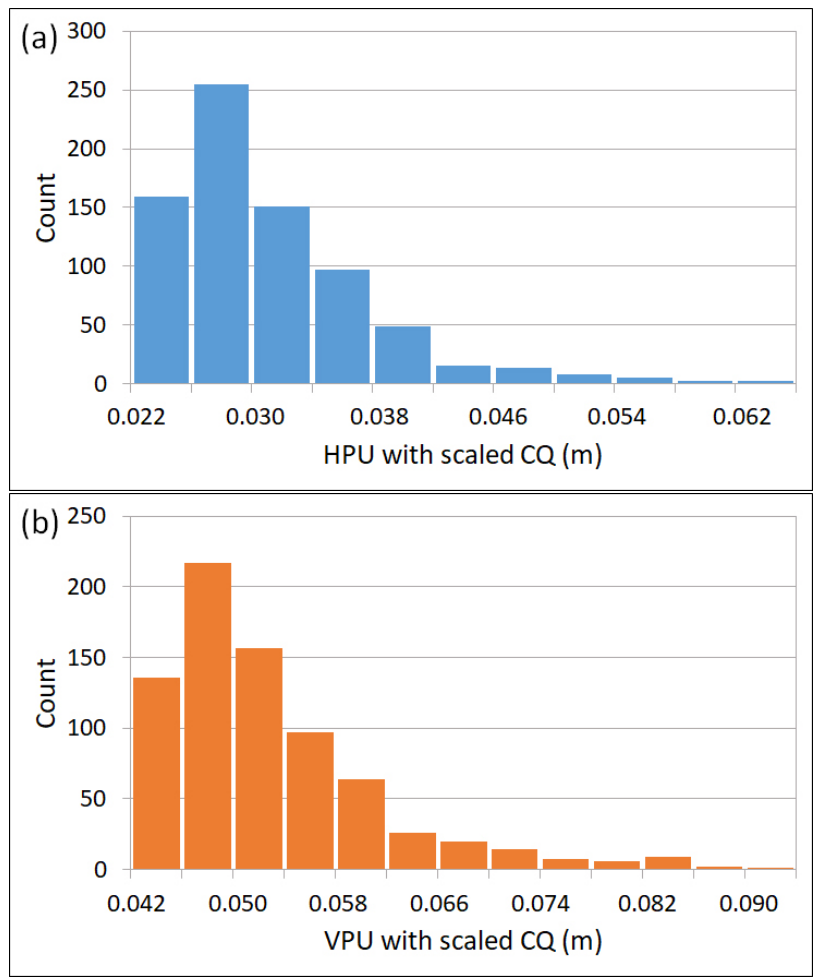

Fig. 5. Calculated (a) HPU and (b) VPU for NRTK observations based on CQ output scaled by a factor of 2.1.

The median values of $0.030 \mathrm{~m}$ (HPU) and $0.051 \mathrm{~m}$ (VPU) are now more closely aligned with the observed quality computed in section 2, indicating that using scaled CQ values in the calculation of PU may be a feasible option for introducing NRTK uncertainties into the GDA2020 state adjustment. However, the scale factor may not be appropriate for all types of GNSS rover equipment due to differences in how CQ is calculated by various equipment manufacturers. Further research would be required in an attempt to determine appropriate values for different receiver brands and models. Furthermore, the potential need to apply separate scale factors for the horizontal and vertical components should be investigated.

In summary, this approach utilises reported CQ values in the calculation of PU, providing calculated PU values rather than universal estimates. While a scale factor can be applied to account for the overly optimistic CQ output in order to obtain more realistic PU values, this does add statistical guesswork to a process that was intended to be a more rigorous alternative to the first option considered. The varying proprietary methods of CQ computation between makes and models of GNSS receivers add further complexity to the derivation of a reliable scale factor.

This method also continues to treat NRTK observations as point-based solutions with uncertainties, thus ex- 
hibiting poor correlation with surrounding marks in the GDA2020 state adjustment, and historical data would have to be reprocessed. Consequently, it does not provide a significant advantage over the use of empirically derived values, while adding a degree of complexity.

\section{Network adjustment using automatic baselines}

NRTK observations are generally treated as point-based solutions with VRS data being discarded after computation, which causes issues when attempting to incorporate NRTK observations and uncertainties into a least squares network adjustment. Therefore, the third option investigated a network solution using the automatically computed baselines from the VRS to each observed station.

Depending on fieldwork practices, multiple observations share a common VRS and are therefore linked by GNSS baselines. A VRS generally remains active until the GNSS rover is turned off or moves more than $5 \mathrm{~km}$ away (Landau et al. 2002), i.e. a typical NRTK survey usually exhibits a high degree of connectivity. These connections potentially allow PU values to be rigorously computed via least squares analysis, facilitating simple integration of NRTK data into the GDA2020 state adjustment.

While the VRS coordinates are computed from surrounding CORS data (with the CORS forming the backbone of the datum), it can be argued that the VRS itself is technically not connected to the datum. However, the VRS can be treated as a pseudo-datum station, with the connection to datum completed by deriving a baseline from each VRS to the nearest (or multiple) CORS. Following the philosophy applied by the Intergovernmental Committee on Surveying and Mapping (ICSM) for including National GNSS Campaign Archive (NGCA) data into the national GDA2020 adjustment, a connection to the two nearest CORS is used here. It is important to note that these derived baselines are not observations, but simply joins used to connect the VRS (and thus the survey) to the datum and to transfer the uncertainty of the datum connection through to the survey network in the adjustment.

As such, this approach employs the automatically computed GNSS baselines between NRTK observations and their VRS together with a derived join between each VRS and the two nearest CORS to create a connected network, which can be adjusted like a traditional, static GNSS network (Fig. 6).

A typical urban NRTK survey incorporating 126 observations on 62 marks in Sydney, conducted by NSW Spa-

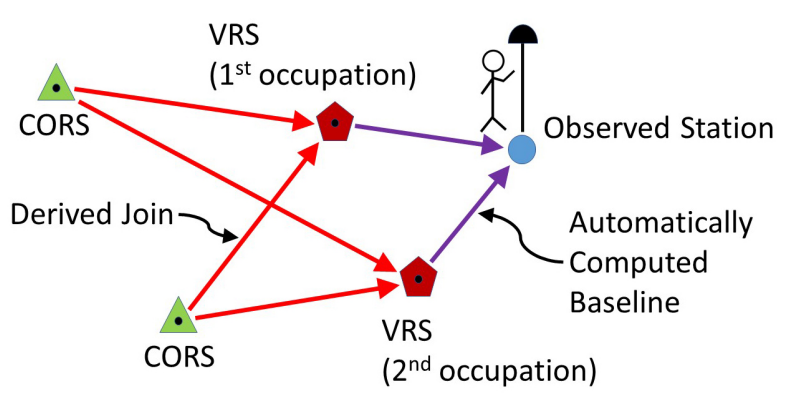

Fig. 6. NRTK automatic baseline network being connected to datum via a join between each VRS and the two nearest CORS.

tial Services over 9 days in 2018, was investigated to illustrate this approach. Best practice guidelines were followed, with each mark occupied at least twice, at least 30 minutes apart, and for a minimum of 2 minutes (ICSM 2020b; NSW Spatial Services 2019). The use of multiple occupations on each mark adds redundancy, strengthens network geometry and helps minimise outliers. The resulting network exhibited a high degree of connectivity through the baselines automatically generated between VRS and observed mark. While the user has limited control over the network geometry created in this way, the network can be processed akin to a static GNSS survey.

In order to perform a least squares adjustment and allow this survey to influence and be influenced by the datum, it must be connected to it. In this case, six control marks that are part of the GDA2020 state adjustment were observed to provide this datum connection, leaving 56 marks to be adjusted. However, considering that a new VRS is generated when the instrument is turned off or moved more than $5 \mathrm{~km}$ from its original VRS location, some marks can potentially become isolated (or disconnected) from the network and datum. In order to ensure connection of all marks to the network, the VRS were treated as pseudo-datum stations joined to the nearest two CORSnetNSW sites, which were then also constrained in the adjustment (Fig. 7).

In order to analyse the statistical results produced by this approach, and to obtain preliminary values of PU, this survey network was adjusted separately to the GDA2020 state adjustment. These PU values will be updated when this network is incorporated into the GDA2020 state adjustment.

The determination of NRTK uncertainty based on modelling the contributing errors is an ongoing area of research (e.g. Baybura et al. 2019; Ouassou and Jensen 2019; Jongrujinan and Satirapod 2020). In this case, baseline weightings were chosen to mimic the standard deviation values $(1 \sigma)$ routinely used by NSW Spatial Ser- 


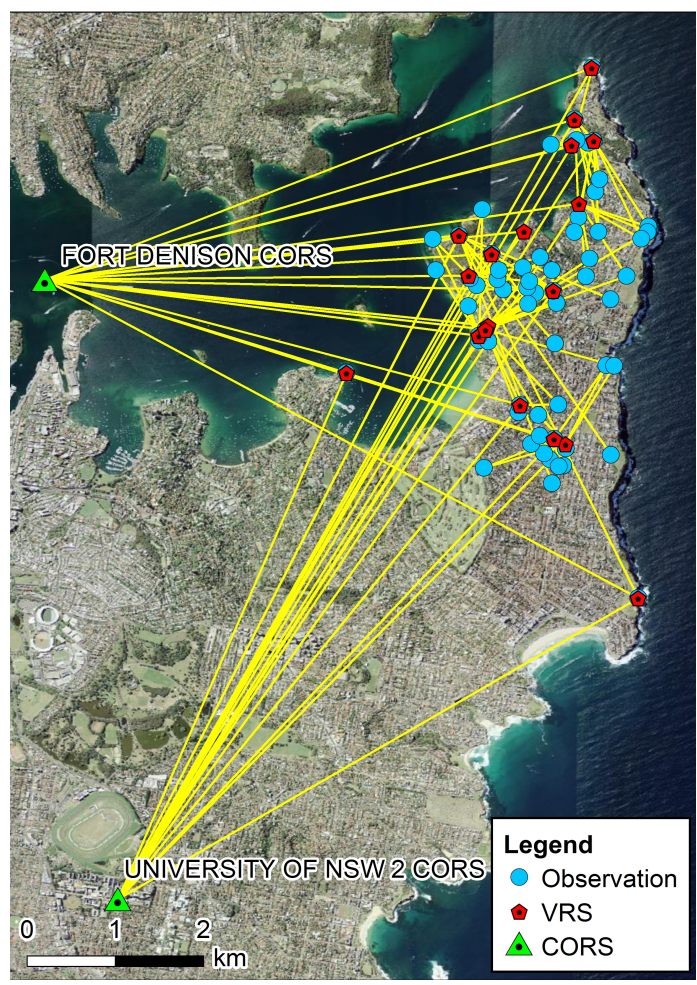

Fig. 7. NRTK baseline network including the joins from each VRS to the two nearest CORS.

vices for NRTK uncertainty in practice: $0.014 \mathrm{~m}$ (horizontal) and $0.030 \mathrm{~m}$ (vertical). These values include allowance for to/from centring errors and have proven realistic in most practical observing conditions using CORSnet-NSW. While it is known that NRTK observations exhibit a small degree of distance dependency, recent studies have found no significant differences in NRTK solutions with baseline lengths of up to 40-50 km to the nearest CORS (Gökdas and Özlüdemir 2020). Consequently, residual NRTK distance dependency can be ignored in this case.

Since each observation is connected to the datum by two baselines (i.e. CORS to VRS and VRS to occupied mark) and to avoid inflation of the uncertainties through this join in the adjustment, these initial values were divided by $\sqrt{ } 2$ according to the law of propagation of errors. This resulted in final weightings of $0.010 \mathrm{~m}$ (horizontal) and $0.021 \mathrm{~m}$ (vertical) for each baseline, with no distance dependency applied.

As previously mentioned, the two CORS served as constraints in the fully constrained adjustment, along with the six GDA2020 control marks that braced the network. The median HPU of these eight constraints was 0.018 $\mathrm{m}$, and the median VPU was $0.026 \mathrm{~m}$. The adjustment achieved a variance factor of 0.9 , which is expected for a network of this nature. In this case, as a business rule,
NSW Spatial Services does not tighten the input standard deviations to achieve a variance factor of unity. Histograms of the resulting PU values are shown in Fig. 8, while corresponding descriptive statistics are summarised in Table 6.
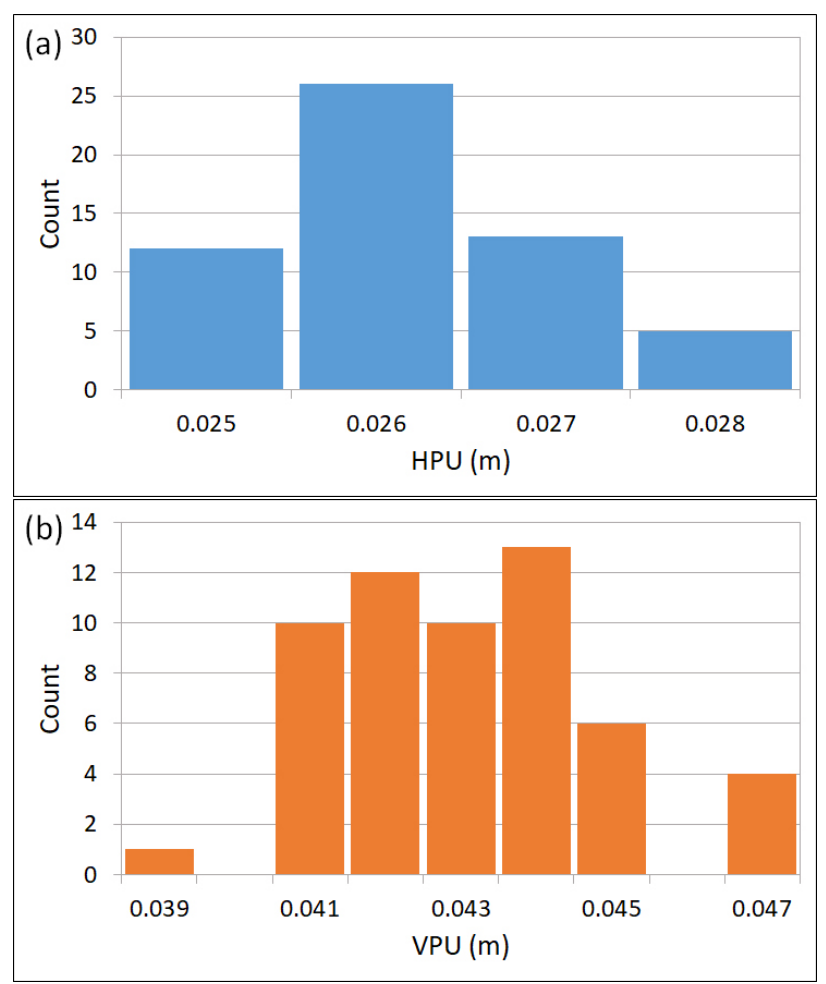

Fig. 8. Calculated (a) HPU and (b) VPU for NRTK observations based on the NRTK baseline network.

Table 6. Descriptive statistics for HPU and VPU, calculated based on the NRTK baseline network for 56 adjusted marks.

\begin{tabular}{ccc}
\hline Descriptive Statistic & HPU $(\mathrm{m})$ & VPU $(\mathrm{m})$ \\
\hline Minimum & 0.025 & 0.039 \\
Maximum & 0.028 & 0.047 \\
Mean & 0.026 & 0.043 \\
Median & 0.026 & 0.043 \\
Standard Deviation & 0.001 & 0.002 \\
\hline
\end{tabular}

The adjustment provided individual uncertainties for each NRTK observation, with median values of $0.026 \mathrm{~m}$ (HPU) and $0.043 \mathrm{~m}$ (VPU), i.e. about $0.010 \mathrm{~m}$ and $0.015 \mathrm{~m}$ better than the empirical estimates obtained in section 2 . This can be explained by improved geometry and redundancy due to the network adjustment. These preliminary results demonstrate the appropriateness of the observa- 
tional weighting strategy used and that this method can provide reliable results.

Of the three options explored to incorporate NRTK observations and uncertainties into the GDA2020 state adjustment, this provides not only the most rigorous method of computing PU, but the baseline data format also allows easy integration into the least squares network adjustment. Furthermore, these benefits come at no expense to fieldwork time or complexity, and the processing load is only marginally increased compared to the traditional NRTK processing methodology followed at NSW Spatial Services, once CORS-to-VRS baseline derivation is automated. NRTK was intended to be a rapid and precise form of positioning, and this method manages to maintain this spirit whilst providing more in-depth statistical analysis and quality reporting. However, one weakness of this method is the difficulty of applying it to historical data, with each NRTK survey needing to be adjusted individually.

\section{Concluding remarks}

Australia's new national datum, GDA2020, is based on a single, nationwide least squares network adjustment that rigorously propagates uncertainty. This paper has investigated three options to include NRTK observations and their Positional Uncertainty in the NSW survey control network via the GDA2020 state adjustment.

In the first option, the PU of NRTK observations was empirically estimated to be $0.036 \mathrm{~m}$ (HPU) and $0.059 \mathrm{~m}$ (VPU), based on a robust historical NRTK dataset of 1,535 observations on 756 marks collected under typical conditions encountered in surveying practice. While this simplistic method can be uniformly applied to future and historical NRTK data, it only provides estimated (rather than rigorously calculated) uncertainties, which may not be realistic under more challenging conditions.

In the second option, the PU was calculated individually for each NRTK observation, based on the coordinate quality indicators provided by the GNSS rover, resulting in overly optimistic values of about $0.023 \mathrm{~m}$ (HPU) and $0.043 \mathrm{~m}$ (VPU). This was mainly attributed to the overly optimistic reporting of CQ values by the GNSS equipment, while also acknowledging the difficulty to reliably estimate the PU of the VRS used. Whilst a scale factor of 2.1 was applied to obtain more realistic CQ values, delivering values of $0.030 \mathrm{~m}$ (HPU) and $0.051 \mathrm{~m}$ (VPU), this added complexity and statistical guesswork to a process that was intended to be more rigorous than the empirically derived PU estimate. Furthermore, any historical data requires reprocessing. Both of these options continue to treat NRTK observations as point-based position solutions, resulting in poor correlation with surrounding survey control marks.

The third option overcomes this issue by handling NRTK data in an entirely different manner. It employs the automatically computed GNSS baselines between NRTK observations and their VRS and derives a join between each VRS and the two nearest CORS to create a connected network, which can be adjusted like a static GNSS network. Using a typical urban NRTK survey incorporating 126 observations on 62 marks in Sydney as an example, PU was calculated to be about $0.026 \mathrm{~m}$ (HPU) and $0.043 \mathrm{~m}$ (VPU), comparing reasonably well to empirical positioning quality and user experience.

This method offers a rigorous computation of PU, while maintaining the quick and easy nature of NRTK positioning. Furthermore, these benefits come at no expense to fieldwork time or complexity, requiring only a slightly more involved processing strategy (network adjustment rather than site transformation) and a tool to generate the derived baselines between CORS and VRS necessary for the join. The need for each historical NRTK survey to be adjusted separately is a reasonable price to pay for this rigorous and comprehensive solution. Adoption of this methodology will allow NRTK data to be rigorously included in the GDA2020 state adjustment, thus enabling NSW Spatial Services to further maintain and extend the NSW survey control network.

\section{References}

Altamimi Z., Rebischung P., Métivier L. and Collilieux X., 2016, ITRF2014: A new release of the International Terrestrial Reference Frame modelling nonlinear station motions, J. Geophys. Res. Solid Earth, 121, 8, 6109-6131.

Baybura T., Tiryakioglu I., Ugur M.A., Solak H.I. and Safak S., 2019, Examining the accuracy of Network RTK and long base RTK methods with repetitive measurements, J. Sens., 2019, 3572605, https://doi.org/10.1155/2019/3572605.

Edwards S.J., Clarke P.J., Penna N.T. and Goebell S., 2010, An examination of Network RTK GPS services in Great Britain, Surv. Rev., 42, 316, 107-121.

Gökdas Ö. and Özlüdemir M.T., 2020, A variance model in NRTK-based geodetic positioning as a function of baseline length, Geosci., 10, 7, 262, https://doi.org/10.3390/ geosciences10070262.

ICSM, 2020a, Geocentric Datum of Australia 2020 technical manual, version 1.5, https://www.icsm.gov.au/gda2020-and-gda94technical-manuals (accessed Jan 2021).

ICSM, 2020b, Standard for the Australian survey control network (SP1), version 2.2, https://www.icsm.gov.au/publications/ 
standard-australian-survey-control-network-specialpublication-1-sp1 (accessed Jan 2021).

Janssen V. and Haasdyk J., 2011, Assessment of Network RTK performance using CORSnet-NSW, Proc. IGNSS Symposium 2011 (IGNSS2011), 15-17 November, Sydney, Australia, 18pp.

Janssen V. and McElroy S., 2020, Assessment of AUSPOS performance in New South Wales, Australia, Proc. IGNSS Symposium 2020 (IGNSS2020), 5-7 February, Sydney, Australia, 17pp.

Janssen V., 2012, Indirect tracking of drop bears using GNSS technology, Aust. Geogr., 43, 4, 445-452.

Janssen V., Gowans N., Hine S. and McElroy S., 2019, Removing the uncertainty surrounding Positional Uncertainty and Local Uncertainty in SCIMS, Azimuth, 58, 8, 28-32.

Janssen V., Haasdyk J. and McElroy S., 2016, CORSnet-NSW: A success story, Proc. Association of Public Authority Surveyors Conference (APAS2016), 4-6 April, Leura, Australia, 10-28.

Jongrujinan T. and Satirapod C., 2020, Stochastic modeling for VRS network-based GNSS RTK with residual interpolation uncertainty, J. Appl. Geod., 14, 3, 317-325.
Landau H., Vollath U. and Chen X., 2002, Virtual reference station systems, J. Global Pos. Sys., 1, 2, 137-143.

NSW Spatial Services, 2019, Surveyor-General's Direction No. 12: Control Surveys and SCIMS, https://www.spatial.nsw.gov.au/ surveying/surveyor_generals_directions (accessed Jan 2021).

NSW Spatial Services, 2021, CORSnet-NSW, http://www.corsnet. com.au/ (accessed Jan 2021).

Ouassou M. and Jensen A.B.O., 2019, Network real-time kinematic data screening by means of multivariate statistical analysis, SN Appl. Sci., 1, 6, 512, https://doi.org/10.1007/s42452-0190531-3.

Queensland Government, 2020, Cadastral survey requirements, version 7.1, reprint 2, Department of Natural Resources, Mines and Energy, Australia, https://www.dnrme.qld.gov.au/_data/ assets/pdf_file/0013/105601/cadastral-survey-requirements. pdf (accessed Jan 2021).

Wang C., Feng Y., Higgins M. and Cowie B., 2010, Assessment of commercial Network RTK user positioning performance over long inter-station distances, J. Global Pos. Sys., 9, 2, 78-89. 